\title{
Salud percibida y rendimiento académico en adolescentes de escuelas públicas de la ciudad de Córdoba
}

\author{
Perceived health and academic performance among adolescents \\ from public schools in the city of Córdoba
}

Mgt. Romina Vitale ${ }^{a}$ Lic. Emilse Degoy y Prof. Dra. Silvina Berra ${ }^{b}$

\begin{abstract}
RESUMEN
Introducción. El desempeño en la escuela podría estar vinculado a la salud en la adolescencia, y los logros educativos en esta edad pueden influir en la vida futura. El objetivo fue estudiar la asociación entre el rendimiento académico y la salud percibida en adolescentes, teniendo en cuenta las características sociodemográficas de sus familias.
\end{abstract}

Población y métodos. Estudio piloto transversal en una muestra de adolescentes asistentes al ciclo básico de tres escuelas secundarias públicas de la ciudad de Córdoba, Argentina. El rendimiento académico se computó como el promedio de las calificaciones en todas las asignaturas y se consideró suficiente cuando fue igual a 6 puntos o mayor. La salud percibida se valoró con el cuestionario KIDSCREEN-52, que produce puntuaciones en diez dimensiones. Además, se recogió la edad, el sexo, el nivel de escolaridad materna, el nivel socioeconómico y la composición de la familia. Se realizaron análisis univariados, bivariados y modelos multivariados de regresión logística.

Resultados. Participaron 554 adolescentes, $52 \%$ de los cuales eran mujeres. El rendimiento académico insuficiente $(27,6 \%)$ fue más frecuente en los adolescentes que manifestaron peor relación con los padres (OR 2,68; IC 95\%: 1,22$5,85)$ y mejor relación con amigos (OR 0,46; IC 95\%: 0,26-0,82). Hubo diferencias al estratificar por nivel socioeconómico: en el nivel alto, el rendimiento insuficiente fue más frecuente en adolescentes con percepción demenor autonomía $\mathrm{y}$, en el nivel medio-bajo, en adolescentes con peor percepción del entorno escolar.

Conclusión. El rendimiento académico se asoció a dimensiones psicosociales de la salud, como sus relaciones familiares, con amigos, autonomía y entorno escolar.

Palabras clave: adolescentes, escolaridad, calidad de vida, salud del niño.

http:/ /dx.doi.org/10.5546/aap.2015.526

\section{INTRODUCCIÓN}

En la perspectiva del ciclo vital, la adolescencia es una etapa de desarrollo marcada por cambios corporales y psicosociales, en la que la salud resulta del estado anterior y condiciona a los siguientes con una sensibilidad y dinámica mayor que en otros momentos. ${ }^{1}$

La salud es un recurso que incluye capacidades personales, sociales, físicas y psicológicas, que permite a las personas afrontar su entorno para realizar sus aspiraciones y satisfacer sus necesidades. ${ }^{2}$ En la infancia y en la adolescencia, el concepto de salud incluye también la habilidad de realizar actividades apropiadas para su edad, la capacidad para las adquisiciones cognitivas, las emociones, la percepción de sí mismo, las relaciones interpersonales y con el medio que los rodea, así como realizar actividades diarias. ${ }^{3}$

El aprendizaje y el rendimiento escolar son funciones primordiales en la infancia. Los logros educativos en esta etapa influyen en la participación laboral y social de las futuras personas adultas. ${ }^{4,5}$ Un indicador importante del proceso de enseñanza-aprendizaje es el rendimiento académico, ${ }^{6}$ definido como "el producto que da el alumnado en los centros de enseñanza y que habitualmente se expresa a través de las calificaciones escolares", ${ }^{\prime}$ a la vez que se lo reconoce influido por factores de índole familiar, socioeconómica, cultural y política. ${ }^{8}$

Actualmente, es posible estudiar la salud de los adolescentes mediante modelos teóricos multidimensionales y teniendo en cuenta su propia perspectiva, ${ }^{9,10}$ e indagar la relación entre la salud y el desempeño escolar en nuestro contexto. En la bibliografía, se encuentran unas pocas investigaciones que vinculan el desempeño en la escuela con 
indicadores de salud, como el bienestar, la satisfacción con la vida ${ }^{11}$ y la salud emocional. ${ }^{12}$ Un estudio realizado en Chile hizo referencia a que los escolares que fracasaban en la escuela habían expresado no estar a gusto con el colegio y se consideraban a sí mismos como peores que el promedio. ${ }^{13}$ Estos antecedentes dan lugar a la hipótesis de que el rendimiento académico estaría asociado a dimensiones psicosociales de la calidad de vida relacionada con la salud (CVRS).

El objetivo de esta investigación fue analizar la asociación entre rendimiento académico promedio anual (RA) y la salud percibida en adolescentes que asistían a escuelas públicas de la ciudad de Córdoba, teniendo en cuenta las características socioeconómicas de sus familias.

\section{POBLACIÓN Y MÉTODOS}

Se realizó un estudio piloto de diseño transversal en adolescentes de ambos sexos asistentes al ciclo básico (de $1^{\circ}$ a $3^{\text {er }}$ año) de tres escuelas secundarias públicas provinciales de la ciudad de Córdoba, cuyos directivos autorizaron la realización de la investigación. El total de escolares matriculados en el ciclo básico era de 862. Se calculó un tamaño muestral de 393 estudiantes como necesario para un contraste bilateral y se aceptó un riesgo alfa de 0,05 y un riesgo beta de 0,2 para detectar una diferencia de 3 o más puntos en el indicador de CVRS. El procedimiento de selección incluyó el envío de un formulario que explicaba los objetivos del estudio y las características generales del instrumento y que solicitaba la firma del consentimiento por parte del tutor. La encuesta se realizó dentro del aula y en dos días hábiles diferentes para que los adolescentes que habían faltado en la primera oportunidad pudieran participar del estudio. El consentimiento informado firmado por los tutores y el asentimiento de los estudiantes fueron requisitos de participación y no se aplicaron otros criterios de exclusión.

El RA se determinó mediante el promedio de las calificaciones escolares del ciclo lectivo, obtenido de los historiales académicos. La calificación media se consideró insuficiente cuando fue menor de 6 puntos y suficiente cuando fue igual a 6 puntos o mayor.

Los adolescentes respondieron un cuestionario que incluyó preguntas sobre su edad, sexo, salud percibida, nivel de escolaridad materna, recursos materiales en el hogar y estructura familiar. Para evaluar la salud percibida, se incluyó el cuestionario KIDSCREEN-52 de
CVRS, que considera 10 dimensiones: bienestar físico, bienestar psicológico, estado de ánimo y emociones, autopercepción, autonomía, relación con los padres y vida familiar, relación con los amigos y apoyo social, entorno escolar, aceptación social, recursos económicos. ${ }^{14} \mathrm{El}$ KIDSCREEN es un instrumento genérico, desarrollado mediante técnicas cualitativas que proporcionaron ítems a partir del discurso de niños, niñas y adolescentes, ${ }^{15}$ con selección de contenido y confirmación de fiabilidad y validez mediante técnicas estadísticas modernas. ${ }^{16}$ Este instrumento fue adaptado transculturalmente para la población argentina de 8-18 años ${ }^{17}$ y sus propiedades métricas fueron confirmadas también en el contexto local. ${ }^{18}$ Las preguntas del cuestionario se responden en una escala Likert con opciones de frecuencia e intensidad que puntúan de 1 a 5 , con lo que se calcula primero un puntaje promedio de las respuestas correspondientes a cada una de las dimensiones; luego se trasforma el puntaje en un conjunto lineal continuo utilizando un modelo RASCH y es estandarizado a una media de 50 y un desvío estándar (DE) de 10 puntos. Finalmente, cada dimensión se categoriza en tres niveles con un punto de corte en $\pm 1 / 2 \mathrm{DE}$, que es bajo: $<45$ puntos; medio: de 45 a 55 puntos; y alto: $>55$ puntos. ${ }^{14}$

La edad escolar es una variable que se calculó para indicar si los adolescentes tenían la edad esperada para el año que cursaban o si tenían una edad mayor.

El nivel de escolaridad materna se clasificó en sin estudios o hasta nivel primario, nivel secundario, nivel terciario o universitario. El nivel socioeconómico (NSE) se indagó a través de la escala de bienestar económico familiar (Family Affluence Scale; FAS, por sus siglas en inglés), ${ }^{19}$ compuesta de preguntas sobre recursos materiales en el hogar (número de autos y computadoras que posee la familia, habitación propia e individual del adolescente y vacaciones familiares en los últimos 12 meses), a partir de la cual se categoriza el puntaje obtenido en NSE bajo, medio y alto. La estructura familiar se consideró nuclear cuando la familia estaba constituida por el padre y la madre convivientes con hijos a su cargo; monoparental, si la familia estaba constituida por uno o varios hijos que tenían a su cabeza un solo progenitor; extensa monoparental, a aquellas familias resultantes de la convivencia de, al menos, tres generaciones en sentido vertical con la presencia de un solo progenitor a cargo de sus hijos; reconstituida, a los núcleos familiares constituidos después de la 
separación de uno de los cónyuges; sin mamá ni papá, cuando la familia estuvo conformada por uno o varios hijos sin sus progenitores a cargo.

La encuesta fue autoadministrada a los alumnos de cada escuela en el aula. Previamente, una persona del equipo explicó los objetivos de la investigación, presentó sus consideraciones éticas e indicó la forma de completar el cuestionario.

\section{Análisis estadístico}

En primer lugar, se realizó un análisis descriptivo univariado de las características de la muestra, calculando media y DE para las variables cuantitativas y distribuciones de frecuencias para las categóricas. Las asociaciones entre variables categóricas se exploraron utilizando el test de Chi cuadrado y las de variables cuantitativas, con el T-test de Student, ambos con un nivel de significación estadística en el valor de $\mathrm{p}<0,05$. Finalmente, se ajustaron modelos de regresión logística multivariada para analizar el efecto independiente de las variables.

En relación con el rendimiento académico, se utilizó la regresión logística múltiple y se estimó mediante OR y sus intervalos de confianza con un nivel del 95\% (IC 95\%). En el modelo multivariado, se incluyeron aquellas variables que presentaron un valor $\mathrm{p} \leq 0,20$ en el análisis bivariado.

\section{Aspectos éticos}

Durante el desarrollo de la investigación, fueron respetadas las recomendaciones internacionales sobre ética y buenas prácticas en la investigación en salud humana. El estudio fue evaluado y aprobado por el Comité Institucional de Ética e Investigación en Salud (CIES) del Sanatorio del Salvador.

\section{RESULTADOS}

La participación fue del $64 \%$ porque respondieron el cuestionario 552 adolescentes de los 862 que integraban la población elegible en las tres escuelas. El principal motivo de no participación fue la falta de consentimiento firmado por los padres (233 casos), seguido de inasistencia (43), falta de consentimiento de los padres (23) o de asentimiento propio (11). En 27 casos, no se obtuvo el rendimiento académico promedio anual porque, en el transcurso del ciclo lectivo, abandonaron la escuela o se trasladaron a otra institución.

El 52\% de los participantes fueron mujeres; el 40,9\% tenía uno o más años por sobre la edad esperada para el año académico en curso. En $21,8 \%$ de los casos, la madre tenía primaria completa o menor escolaridad; y el 37,9\% de los adolescentes convivía en una familia diferente de la nuclear (Tabla 1).

TABla 1. Características de la muestra según sexo

\begin{tabular}{|c|c|c|c|}
\hline & Mujeres & Varones & Total \\
\hline Sexo, \% (n) & $265(52 \%)$ & $287(48 \%)$ & 552 \\
\hline Edad (DE) & $14,1(1,3)$ & $13,8(1,3)$ & $13,9(1,3)$ \\
\hline $\begin{array}{l}\text { Edad escolar, \% (n) } \\
\text { Edad teórica } \\
1 \text { año más de lo esperado } \\
2 \text { o más años de lo esperado }\end{array}$ & $\begin{array}{l}59,5 \%(153) \\
22,2 \%(57) \\
18,3 \%(47)\end{array}$ & $\begin{array}{l}58,7 \%(168) \\
24,8 \%(71) \\
16,4 \%(47)\end{array}$ & $\begin{array}{c}59,1 \%(321) \\
23,6 \%(128) \\
17,3 \%(94)\end{array}$ \\
\hline $\begin{array}{l}\text { Escolaridad materna, } \% \text { (n) } \\
\text { Terciario o universitario } \\
\text { Secundario } \\
\text { Primario completo o menos }\end{array}$ & $\begin{array}{c}28,0 \%(67) \\
46,4 \%(111) \\
25,5 \%(61)\end{array}$ & $\begin{array}{c}31,3 \%(79) \\
50,4 \%(127) \\
18,3 \%(46)\end{array}$ & $\begin{array}{l}29,7 \%(146) \\
48,5 \%(238) \\
21,8 \%(107)\end{array}$ \\
\hline $\begin{array}{l}\text { Nivel socioeconómico (FAS), \% } \\
\text { Bajo } \\
\text { Medio } \\
\text { Alto }\end{array}$ & $\begin{array}{c}27 \%(70) \\
43,4 \%(112) \\
29,5 \%(76)\end{array}$ & $\begin{array}{c}19,2 \%(53) \\
47,8 \%(132) \\
33 \%(91)\end{array}$ & $\begin{array}{c}23 \%(123) \\
45,7 \%(244) \\
31,3 \%(167)\end{array}$ \\
\hline $\begin{array}{l}\text { Estructura familiar, \% (n) } \\
\text { Nuclear } \\
\text { Monoparental } \\
\text { Extensa monoparental } \\
\text { Reconstituida } \\
\text { Sin mamá ni papá }\end{array}$ & $\begin{array}{c}57,0 \%(151) \\
25,3 \%(67) \\
2,6 \%(7) \\
12,1 \%(32) \\
3 \%(8)\end{array}$ & $\begin{array}{c}66,8 \%(191) \\
13,6 \%(39) \\
4,9 \%(14) \\
11,5 \%(33) \\
3,1 \%(9)\end{array}$ & $\begin{array}{c}62,1 \%(342) \\
19,2 \%(106) \\
3,8 \%(21) \\
11,8 \%(65) \\
3,1 \%(17)\end{array}$ \\
\hline
\end{tabular}

FAS: escala de bienestar económico familiar. 
El RA fue de 6,61 puntos (DE 1,1). El 72,4\% de los adolescentes calificaron con 6 o más y conformaron el grupo de RA suficiente. En el análisis bivariado, el RA insuficiente estuvo asociado al sexo masculino $(p=0,01)$, a una edad mayor a la esperada $(p=0,01)$ y fue más frecuente en adolescentes sin madre ni padre $(p=0,04)$ (Figura 1), mientras que no se confirmó una asociación con el NSE y la escolaridad materna.

Las dimensiones de la CVRS asociadas al RA en el análisis bivariado fueron el estado de ánimo, la relación con los padres y el entorno escolar, en las que tuvieron puntuaciones promedio más bajas los adolescentes con RA menor de 6 (insuficiente) frente a aquellos con RA suficiente (Tabla 2). El porcentaje de adolescentes con malestar en la dimensión "entorno escolar" (puntuaciones $<45$ en la escala de KIDSCREEN) fue mayor en los que tuvieron rendimiento insuficiente $(61,2 \%)$ que en los adolescentes con promedio mayor a seis $(40,1 \%)$.

En el análisis multivariado, se confirmó que el RA insuficiente fue más frecuente en adolescentes varones ( $\mathrm{OR}_{\mathrm{a}} 1,75$; IC 95\%: 1,12-2,73) en comparación con las mujeres, en los que tenían un año (OR a,12; IC 95\%: 1,70-5,71) o dos años o más de la edad esperada $\left(\mathrm{OR}_{\mathrm{a}} 2,71\right.$; IC 95\%: 1,35$5,42)$ comparados con aquellos adolescentes con la edad teórica para el año que cursaban, en los adolescentes con peor percepción en la relación con sus padres $\left(\mathrm{OR}_{\mathrm{a}} 2,68\right.$; IC 95\%: 1,22-5,5) y en la categoría intermedia de la dimensión amigos y apoyo social (OR 0,46 ; IC 95\%: 0,26-0,82) comparados con aquellos que puntuaron alto en estas dimensiones (Tabla 3).

Al estratificar la muestra por NSE, se observó que la diferencia de RA por edad escolar se confirmó solo en los adolescentes de NSE alto, y el RA insuficiente fue más frecuente entre los adolescentes con un año (53,3\%, OR a 7,52; IC 95\%: $2,01-28,13)$ y dos años o más de edad mayor a la esperada (66,7\%, OR a 10,60; IC 95\%: 2,10-53,51) (Tabla 4).

La declaración de menor autonomía se asoció a una menor frecuencia de RA insuficiente en adolescentes de NSE medio-bajo (OR 0,35 ; IC $95 \%: 0,15-0,80)$ y a una mayor frecuencia en el NSE alto (OR 3,89; IC 95\%: 1,03-14,63). En los adolescentes de NSE medio-bajo, el RA insuficiente fue más frecuente en aquellos que declararon baja percepción en "entorno escolar" (OR a 3,13; IC 95\%: 1,11-8,82).

FIGURA 1. Frecuencia de rendimiento académico promedio anual suficiente o insuficiente según sexo, edad escolar y composición de la familia

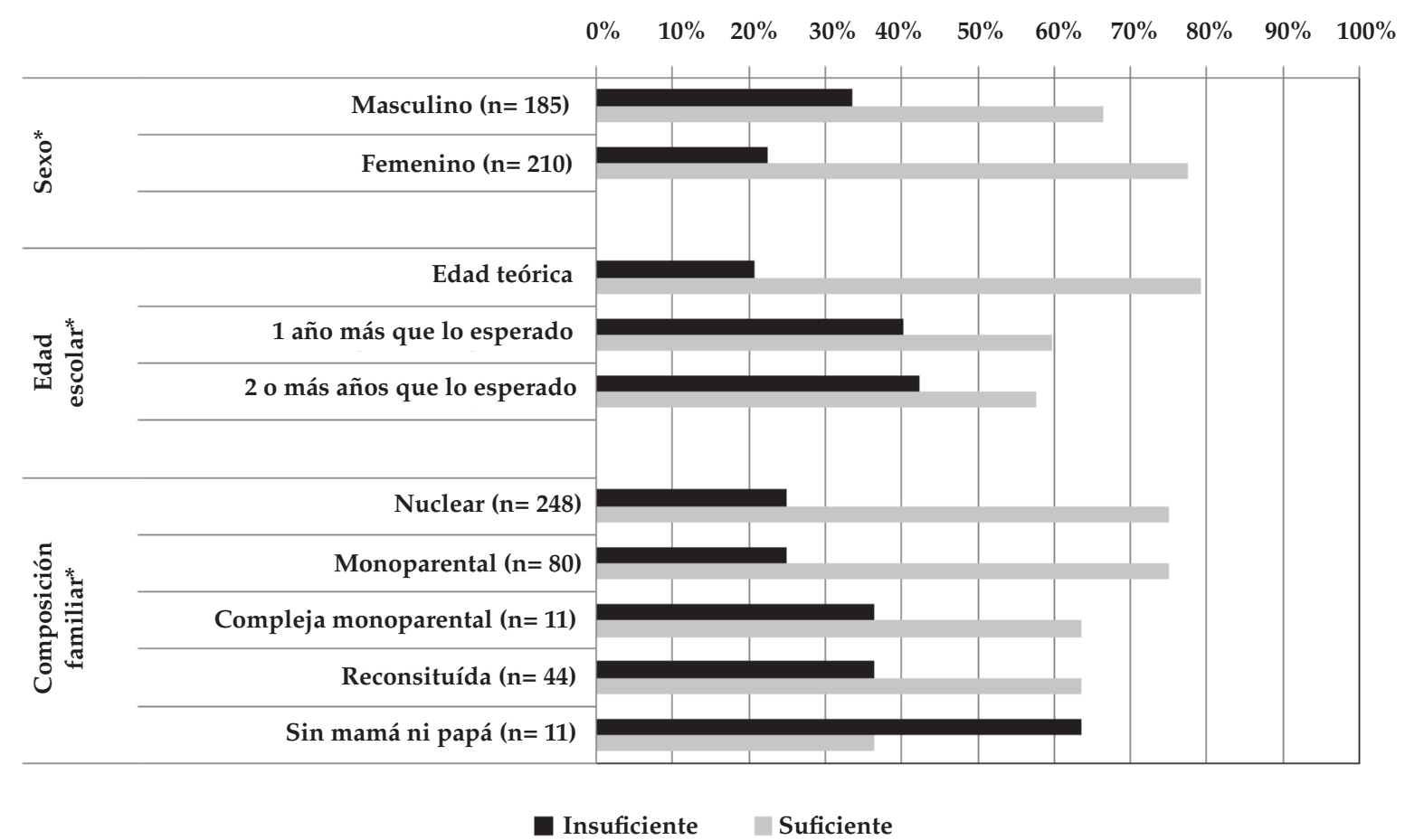

* Diferencias estadísticamente significativas $\left(\mathrm{Chi}^{2} p<0,05\right)$ de la distribución del rendimiento académico en cada variable. 


\section{DISCUSIÓN}

Este trabajo analiza la relación entre la salud y el desempeño escolar en la adolescencia y es, hasta donde conocemos, el primer estudio que aborda este tema en el ámbito de la pediatría en Argentina. La adolescencia representa un período del desarrollo de gran vulnerabilidad, en el que las evidencias disponibles indican que hay un empeoramiento de la salud autopercibida ${ }^{20,21} \mathrm{y}$ del desempeño escolar. ${ }^{11}$ De este modo, se trata de un período crítico que debe atenderse desde distintas disciplinas, incluida la pediatría, ya que la salud y los logros educativos de esta etapa producen profundos efectos sobre la salud y la vida productiva adulta. ${ }^{5}$

Los resultados muestran que el bajo RA se asoció a malestar en dimensiones de la salud, tales como la autonomía, las relaciones con amigos, los sentimientos en el entorno familiar y las percepciones sobre el entorno escolar. Mientras que investigaciones previas realizadas en otros países coinciden con estos resultados al mostrar que, en la infancia, además de algunos indicadores clásicos de morbilidad, el bienestar físico, el estrés, la satisfacción con la vida ${ }^{22-11}$ y la salud emocional ${ }^{12}$ se relacionaron con el desempeño en la escuela, en la adolescencia, según nuestros hallazgos, parecen cobrar más importancia las dimensiones sociales de la salud. Otro estudio realizado en EE.UU., mostró que distintos aspectos de la salud, tal y como sentimientos de vitalidad, bienestar psicológico, conexión con los demás, una baja exposición al hostigamiento (bullying) y la ausencia de estrés, tienen efectos positivos en el desempeño académico, por lo que se concluye que quienes presentan una mejor salud obtienen mejores resultados escolares. ${ }^{11}$

Los adolescentes se enfrentan a la dualidad de dedicar mayor tiempo a las metas académicas o a las metas sociales ${ }^{23}$ y aparece una mayor aprobación hacia los compañeros que hacia el colegio. ${ }^{24}$ En este estudio, los adolescentes que manifestaron una relación con amigos más fuerte tuvieron bajo RA con mayor frecuencia. La autonomía de los adolescentes se asoció al

TABla 2. Puntuaciones de las dimensiones de la calidad de vida relacionada con la salud según el nivel de rendimiento académico en adolescentes escolarizados de la ciudad de Córdoba, 2010

\begin{tabular}{|c|c|c|c|c|c|c|c|c|c|}
\hline \multirow[b]{2}{*}{ CVRS } & \multicolumn{4}{|c|}{ Rendimiento académico insuficiente } & \multicolumn{4}{|c|}{ Rendimiento académico suficiente } & \multirow{2}{*}{$\begin{array}{c}\text { Valor } \mathrm{p} \\
\mathrm{Chi}^{2}\end{array}$} \\
\hline & $\begin{array}{c}\text { Media } \\
\text { (DE) }\end{array}$ & $\begin{array}{c}\% \text { bajo } \\
\text { (n) }\end{array}$ & $\begin{array}{l}\% \text { medio } \\
\text { (n) }\end{array}$ & $\begin{array}{c}\% \text { alto } \\
\text { (n) }\end{array}$ & $\begin{array}{c}\text { Media } \\
\text { (DE) }\end{array}$ & $\begin{array}{c}\% \text { bajo } \\
\text { (n) }\end{array}$ & $\begin{array}{l}\% \text { medio } \\
\text { (n) }\end{array}$ & $\begin{array}{c}\% \text { alto } \\
\text { (n) }\end{array}$ & \\
\hline $\begin{array}{l}\text { Bienestar } \\
\text { físico }\end{array}$ & $\begin{array}{c}46,5 \\
(10,0)\end{array}$ & $\begin{array}{c}49,5 \% \\
(51)\end{array}$ & $\begin{array}{c}35,0 \% \\
(36)\end{array}$ & $\begin{array}{c}15,5 \% \\
(16)\end{array}$ & $\begin{array}{c}48,1 \\
(15,0)\end{array}$ & $\begin{array}{c}45,5 \% \\
(125)\end{array}$ & $\begin{array}{c}31,3 \% \\
(86)\end{array}$ & $\begin{array}{c}23,3 \% \\
(64)\end{array}$ & 0,237 \\
\hline $\begin{array}{l}\text { Bienestar } \\
\text { psicológico }\end{array}$ & $\begin{array}{c}51,6 \\
(10,1)\end{array}$ & $\begin{array}{c}24,5 \% \\
(26)\end{array}$ & $\begin{array}{c}46,2 \% \\
(49)\end{array}$ & $\begin{array}{c}29,2 \% \\
(31)\end{array}$ & $\begin{array}{l}51,6 \\
(9,2)\end{array}$ & $\begin{array}{c}23,0 \% \\
(65)\end{array}$ & $\begin{array}{c}46,6 \% \\
(132)\end{array}$ & $\begin{array}{c}30,4 \% \\
(86)\end{array}$ & 0,940 \\
\hline $\begin{array}{l}\text { Estado } \\
\text { de ánimo }\end{array}$ & $\begin{array}{c}43,4 \\
(10,5)^{*}\end{array}$ & $\begin{array}{c}59,4 \% \\
(63)\end{array}$ & $\begin{array}{c}31,1 \% \\
(33)\end{array}$ & $\begin{array}{c}9,4 \% \\
(10)\end{array}$ & $\begin{array}{c}46,2 \\
(9,9)^{*}\end{array}$ & $\begin{array}{c}51,1 \% \\
(140)\end{array}$ & $\begin{array}{c}32,5 \% \\
(89)\end{array}$ & $\begin{array}{c}16,4 \% \\
(45)\end{array}$ & 0,146 \\
\hline Autopercepción & $\begin{array}{c}50,9 \\
(10,5)\end{array}$ & $\begin{array}{c}28,8 \% \\
(30)\end{array}$ & $\begin{array}{c}40,4 \% \\
(42)\end{array}$ & $\begin{array}{c}30,8 \% \\
(32)\end{array}$ & $\begin{array}{l}50,1 \\
(9,1)\end{array}$ & $\begin{array}{c}31,3 \% \\
(87)\end{array}$ & $\begin{array}{c}42,1 \% \\
(117)\end{array}$ & $\begin{array}{c}26,6 \% \\
(74)\end{array}$ & 0,729 \\
\hline Autonomía & $\begin{array}{c}50,5 \\
(10,3)\end{array}$ & $\begin{array}{c}24,3 \% \\
(25)\end{array}$ & $\begin{array}{c}45,0 \% \\
(46)\end{array}$ & $\begin{array}{c}31,1 \% \\
(32)\end{array}$ & $\begin{array}{l}50,2 \\
(9,4)\end{array}$ & $\begin{array}{c}27,7 \% \\
(77)\end{array}$ & $\begin{array}{c}45,0 \% \\
(124)\end{array}$ & $\begin{array}{c}27,7 \% \\
(77)\end{array}$ & 0,729 \\
\hline $\begin{array}{l}\text { Relación con } \\
\text { los padres y } \\
\text { vida familiar }\end{array}$ & $\begin{array}{c}46,1 \\
(10,2)^{*}\end{array}$ & $\begin{array}{c}43,9 \% \\
(43)\end{array}$ & $\begin{array}{c}40,8 \% \\
(40)\end{array}$ & $\begin{array}{c}15,3 \% \\
(15)\end{array}$ & $\begin{array}{c}49,0 \\
(10,3)^{*}\end{array}$ & $\begin{array}{c}34,4 \% \\
(96)\end{array}$ & $\begin{array}{c}41,9 \% \\
(117)\end{array}$ & $\begin{array}{c}23,7 \% \\
(66)\end{array}$ & 0,114 \\
\hline $\begin{array}{l}\text { Amigos y } \\
\text { apoyo social }\end{array}$ & $\begin{array}{c}54,8 \\
(10,4)\end{array}$ & $\begin{array}{c}18,1 \% \\
(19)\end{array}$ & $\begin{array}{c}36,2 \% \\
(38)\end{array}$ & $\begin{array}{c}45,7 \% \\
(48)\end{array}$ & $\begin{array}{c}54,4 \\
(10,3)\end{array}$ & $\begin{array}{c}13,9 \% \\
(38)\end{array}$ & $\begin{array}{c}47,4 \% \\
(130)\end{array}$ & $\begin{array}{c}38,7 \% \\
(106)\end{array}$ & 0,154 \\
\hline $\begin{array}{l}\text { Entorno } \\
\text { escolar }\end{array}$ & $\begin{array}{c}43,5 \\
(8,4)^{*}\end{array}$ & $\begin{array}{l}61,2 \% \\
(63)^{* *}\end{array}$ & $\begin{array}{c}28,2 \% \\
(29)\end{array}$ & $\begin{array}{c}10,7 \% \\
(11)\end{array}$ & $\begin{array}{c}47,7 \\
(8,7)^{*}\end{array}$ & $\begin{array}{l}40,1 \% \\
(111)^{* *}\end{array}$ & $\begin{array}{c}44,0 \% \\
(122)\end{array}$ & $\begin{array}{c}15,9 \% \\
(33)\end{array}$ & 0,002 \\
\hline $\begin{array}{l}\text { Aceptación } \\
\text { social }\end{array}$ & $\begin{array}{c}44,6 \\
(11,6)\end{array}$ & $\begin{array}{c}57,9 \% \\
(62)\end{array}$ & $\begin{array}{c}10,3 \% \\
(11)\end{array}$ & $\begin{array}{c}31,8 \% \\
(34)\end{array}$ & $\begin{array}{c}45,1 \\
(10,3)\end{array}$ & $\begin{array}{c}53,4 \% \\
(150)\end{array}$ & $\begin{array}{c}18,9 \% \\
(53)\end{array}$ & $\begin{array}{c}27,8 \% \\
(78)\end{array}$ & 0,113 \\
\hline $\begin{array}{l}\text { Recursos } \\
\text { económicos }\end{array}$ & $\begin{array}{l}46,4 \\
(9,2)\end{array}$ & $\begin{array}{c}49,5 \% \\
(54)\end{array}$ & $\begin{array}{c}31,2 \% \\
(34)\end{array}$ & $\begin{array}{c}19,3 \% \\
(21)\end{array}$ & $\begin{array}{l}46,4 \\
(8,3)\end{array}$ & $\begin{array}{c}45,2 \% \\
(128)\end{array}$ & $\begin{array}{c}37,5 \% \\
(106)\end{array}$ & $\begin{array}{c}17,3 \% \\
(49)\end{array}$ & 0,538 \\
\hline
\end{tabular}

DE: desvío estándar; CVRS: calidad de vida relacionada con la salud.

* Diferencias estadísticamente significativas (ANOVA $p<0,05$ ) de las medias de las dimensiones de CVRS según rendimiento académico.

** Diferencias estadísticamente significativas $\left(\mathrm{Chi}^{2} p<0,05\right)$ en las distribuciones de las puntuaciones en las dimensiones de CVRS según rendimiento académico. 
TABLA 3. Variables relacionadas con el rendimiento académico insuficiente en adolescentes escolarizados

\begin{tabular}{|c|c|c|c|}
\hline Variables & $\% *$ & $\mathrm{OR}_{\mathrm{c}}(\mathrm{IC} 95 \%)^{*}$ & $\mathrm{OR}_{\mathrm{a}}(\mathrm{IC} 95 \%)^{* *}$ \\
\hline \multicolumn{4}{|l|}{ Sexo* } \\
\hline Femenino & 22,4 & 1 & 1 \\
\hline Masculino & 33,5 & $1,75(1,12-2,73)$ & $2,28(1,35-3,83)$ \\
\hline \multicolumn{4}{|l|}{ Edad escolar* } \\
\hline Teórica & 20,8 & 1 & 1 \\
\hline 1 año más de lo esperado & 40,3 & $2,56(1,48-4,41)$ & $3,12(1,70-5,71)$ \\
\hline 2 o más años de lo esperado & 42,3 & $2,78(1,49-5,21)$ & $2,71(1,35-5,42)$ \\
\hline \multicolumn{4}{|l|}{ Estructura familiar* } \\
\hline Nuclear & 25,0 & 1 & $\mathrm{NS}^{+}$ \\
\hline Monoparental & 25,0 & $1(0,56-1,79)$ & \\
\hline Compleja monoparental & 36,4 & $1,71(0,49-6,05)$ & \\
\hline Reconstituida & 36,4 & $1,71(0,87-3,38)$ & \\
\hline Sin mamá ni papá & 63,6 & $5,25(1,49-18,54)$ & \\
\hline \multicolumn{4}{|l|}{ CVRS } \\
\hline \multicolumn{4}{|l|}{ Relación con los padres } \\
\hline Alto & 18,5 & 1 & \\
\hline Medio & 25,5 & $1,97(1,01-3,84)$ & $1,85(0,88-3,86)$ \\
\hline Bajo & 30,9 & $1,50(0,77-2,93)$ & $2,68(1,22-5,85)$ \\
\hline \multicolumn{4}{|l|}{ Amigos y apoyo social } \\
\hline Alto & 31,2 & 1 & 1 \\
\hline Medio & 22,6 & $0,65(0,39-1,06)$ & $0,46(0,26-0,82)$ \\
\hline Bajo & 33,3 & $1,10(0,58-2,11)$ & $0,63(0,29-1,38)$ \\
\hline \multicolumn{4}{|l|}{ Entorno escolar } \\
\hline Alto & 20,0 & 1 & NS \\
\hline Medio & 19,2 & $0,95(0,44-2,06)$ & \\
\hline Bajo & 36,2 & $2,27(1,10-4,71)$ & \\
\hline
\end{tabular}

CVRS: calidad de vida relacionada con la salud.

* Odds ratios crudas obtenidas por regresión logística bivariada.

** Odds ratios ajustadas obtenidas por regresión logística multivariada, que incluyen todas las variables presentadas en la tabla.

+ Variables excluidas del modelo multivariado al comprobar que no aportaban contribución significativa. NS: no significativo.

TABLA 4. Factores asociados al rendimiento académico promedio anual insuficiente estratificado por nivel socioeconómico

\begin{tabular}{|c|c|c|c|c|c|c|}
\hline & \multicolumn{3}{|c|}{ NSE medio-bajo $(n=242)$} & \multicolumn{3}{|c|}{ NSE alto $(n=108)$} \\
\hline & $\%$ & $\mathrm{OR}_{\mathrm{c}}(\mathrm{IC}$ 95\%)* & $\mathrm{OR}_{\mathrm{a}}(\mathrm{IC} 95 \%)^{* *}$ & $\%$ & $\mathrm{OR}_{\mathrm{c}}(\mathrm{IC} 95 \%)^{*}$ & $\mathrm{OR}_{\mathrm{a}}(\mathrm{IC} 95 \%)^{* *}$ \\
\hline \multicolumn{7}{|l|}{ Edad escolar } \\
\hline Edad teórica & $20,6^{+}$ & 1 & & $19,3^{+}$ & & \\
\hline 1 año más de lo esperado & 37,1 & $2,27(1,20-4,30)$ & $2,03(1,00-4,09)$ & 53,3 & $4,77(1,52-14,99)$ & $7,52(2,01-28,13)$ \\
\hline 2 o más años de lo esperado & 31,4 & $1,77(0,79-3,96)$ & $1,34(0,55-3,27)$ & 66,7 & $8,35(2,25-31,01)$ & $10,60(2,10-53,51)$ \\
\hline \multicolumn{7}{|l|}{ CVRS } \\
\hline \multicolumn{7}{|l|}{ Autonomía } \\
\hline Alto & 37,8 & 1 & & 37,5 & & \\
\hline Medio & 21,5 & $0,53(0,27-1,03)$ & $0,46(0,22-0,98)$ & 32,1 & $3,27(1,09-9,79)$ & $3,89(1,03-14,63)$ \\
\hline Bajo & 28,0 & $0,45(0,21-0,96)$ & $0,35(0,15-0,80)$ & 16,0 & $3,99(1,13-14,08)$ & $4,17(0,95-18,31)$ \\
\hline \multicolumn{7}{|l|}{ Entorno escolar } \\
\hline Alto & 5,3 & 1 & & $46,4^{+}$ & & \\
\hline Medio & 22,9 & $1,26(0,46-3,41)$ & $1,07(0,37-3,11)$ & 20,8 & $0,69(0,18-2,69)$ & $0,81(0,15-4,41)$ \\
\hline Bajo & $41,9^{+}$ & $2,99(1,16-7,73)$ & $3,13(1,11-8,82)$ & 20,0 & $1,64(0,46-5,82)$ & $1,71(0,34-8,51)$ \\
\hline
\end{tabular}

NSE: nivel socioeconómico; CVRS: calidad de vida relacionada con la salud.

* Odds ratios crudas obtenidas por regresión logística bivariada. ** Odds ratios ajustadas obtenidas por regresión logística multivariada, que incluyen todas las variables presentadas en la tabla. ${ }^{\dagger}$ Diferencias estadísticamente significativas $\left(\mathrm{Chi}^{2} \mathrm{p}<0,05\right)$ de la distribución del rendimiento académico en cada variable, dentro del grupo de NSE bajo-medio o del grupo de NSE alto. 
RA en sentido diferente según el NSE, lo que muestra una asociación positiva en los grupos de NSE alto, pero negativa en los adolescentes de NSE bajo. La interpretación de estos resultados está limitada al hecho de haberse estudiado un grupo sociocultural acotado que merecería una profundización en la comprensión de los significados de las relaciones personales y los logros académicos.

El vínculo con los padres juega un rol importante en el proceso de enseñanza y aprendizaje de los escolares y el ambiente escolar también puede favorecer mejores logros en el aprendizaje. ${ }^{25}$ Los adolescentes que convivían en familias no convencionales obtuvieron calificaciones más bajas que aquellos que convivían con su papá y su mamá, aunque esta asociación no se confirmó en los modelos multivariados, posiblemente debido al tamaño de la muestra. Algunos autores afirman que los niños que conviven en una estructura familiar nuclear podrían conseguir mayores destrezas emocionales y sociales, que permiten un rendimiento académico satisfactorio. ${ }^{26}$ En cuanto a la salud percibida, los adolescentes con bajas calificaciones mostraron peores percepciones de sus relaciones con los padres y en el entorno escolar. El efecto del entorno escolar fue mayor en los jóvenes de NSE bajo.

Si bien el tamaño de la muestra fue suficiente para corroborar como estadísticamente significativas las mayores diferencias halladas, la alta proporción de escolares que no participaron debido a que sus padres no respondieron a la solicitud de consentimiento firmado podría introducir un riesgo de sesgo si existiera un factor asociado tanto al motivo de no participación como al rendimiento académico y a la CVRS.

Otra limitación de este estudio es la imposibilidad de establecer relaciones causales entre los fenómenos estudiados por su diseño transversal. Futuras investigaciones longitudinales podrían dar cuenta del efecto de la salud y las variables sociodemográficas sobre el RA de los adolescentes. Se podría postular la hipótesis de que una buena salud es requisito para el aprendizaje y un rendimiento académico óptimos, pero también se sugiere una relación bidireccional. ${ }^{11,12} \mathrm{Si}$ bien aún existen escasas evidencias acerca del impacto del rendimiento académico sobre la salud percibida de los más jóvenes, algunos estudios ${ }^{27,28}$ sugieren que los adolescentes que reportan mejores calificaciones son más propensos a informar una mejor salud percibida, por lo que el estudio longitudinal de estas variables permitiría dilucidar el tipo de relación y la dinámica que existe entre ellas.

Entre las fortalezas de este trabajo, se debe señalar que se analizaron dos aspectos relevantes en la vida de los adolescentes, como son la educación y la salud, esta última desde su propia perspectiva. A partir de este estudio piloto, es interesante recomendar la profundización de estos enfoques de la investigación pediátrica para comprender otras aristas de la salud en la adolescencia.

\section{CONCLUSIÓN}

Esta investigación muestra la relación entre el desempeño escolar de los adolescentes y su salud autopercibida, y sugiere que dimensiones psicosociales de la CVRS, como las relaciones familiares y con amigos, la autonomía y las percepciones del entorno escolar, son factores primordiales en el análisis de los bajos rendimientos en esta edad, con influencia también de la edad escolar, el género y el NSE.

\section{Agradecimientos}

A las autoridades, personal docente y estudiantes de las escuelas que participaron del estudio. Al equipo de investigación del Centro de Investigación Epidemiológica y en Servicios de Salud y, en particular, a la Mgter. Verónica Mamondi por su colaboración en la coordinación y ejecución del trabajo de campo. A María Eugenia Esandi y Mariana Roizen, que leyeron una versión previa de este manuscrito e hicieron aportes importantes para mejorar su contenido.

\section{REFERENCIAS}

1. National Research Council and Institute of Medicine. Committee on Evaluation of Children's Health Board on Children, Youth and Families, Division of Behavioral and Social Sciences and Education. Children's Health, the Nation's Wealth: Assessing and Improving Child Health. Washington, DC: National Academies Press; 2004.

2. Starfield B. Basic concepts in population health and health care. J Epidemiol Community Health 2001;55(7):452-4.

3. Starfield B. Measurement of outcome: a proposed scheme. Milbank Mem Fund Q Health Soc 1974;52(1):39-50.

4. Rajmil L, Díez E, Peiró R. Desigualdades sociales en la salud infantil. Informe SESPAS 2010. Gac Sanit 2010;24(1):42-8.

5. Melchior M, Moffit TE, Milne BJ, Poulton R, Caspi A. Why do children from socioeconomically disad vantaged families suffer from poor health when they reach adulthood? A lifecousestudy. Am J Epidemiol 2007;166(8):966-74.

6. Edel Navarro R. El rendimiento académico: concepto, investigación y desarrollo. REICE 2003;1(2):1-15.

7. Martínez Carpio H. Autopercepción social y atribuciones cognoscitivas en estudiantes de bajo rendimiento 
académico. Rev Electrón Investig Psicoeduc Psigopedag 2009;7(3):1175-1216.

8. Acero Abdul G, Talman Peluha A, Vergara Galván A, Moreno Méndez J,Jiménez Martín H. Relación entrehábitos de salud, consumo de internet y rendimiento académico en adolescentes de un colegio de la ciudad de Bogotá. Psicogente 2008;11(19):9-23.

9. Solans M, Pane S, Estrada M, Serra-Suton V, et al. Healthrelated quality of life measurement in children and adolescents: A systematic review of generic and diseasespecific instruments. Value Health 2008;11(4):742-64.

10. Rajmil L, Roizen M, Psy AU, Hidalgo-Rasmussen C, et al. Health-related quality of life measurement in children and adolescents in Ibero-American countries, 2000 to 2010. Value Health 2012;15(2):312-22.

11. Forrest CB, Bevans KB, Riley AW, Crespo R, Louis, TA. Health and school outcomes during children's transition into adolescence. J Adolesc Health 2013;52(2):186-94.

12. Ickovics JR, Carroll-Scott A, Peters SM, Schwartz M, et al. Health and academic achievement: cumulative effects of health assets on standardized test scores among urban youth in the United States. J Sch Health 2014;84(1):40-8.

13. Ojembarrena Martínez E, Fernández de Pinedo Montoya R, Gorostiza Garay E, LafuenteMesanza P, Lizarraga Azparren MA. Fracasoescolar en adolescentes y factores sociosanitarios asociados. An Esp Pediatr 2002;56(5):416-424.

14. KIDSCREEN Group Europe. The KIDSCREEN Questionnaires. Quality of Life Questionnaires for Children and Adolescents Handbook. Lengerich: Papst; 2006.

15. Detmar S, Bruil J, Ravens-Sieberer U, Gosch A, Bisegger C; The European KIDSCREEN group. The Use of Focus Groups in the Development of the KIDSCREEN HRQL Questionnaire. Qual of Life Res 2006;15(8):1345-53.

16. Ravens-Sieberer U, Gosch A, Rajmil L, Erhart M, et al. The KIDSCREEN-52 quality of life measure for children and adolescents: psychometric results from a crosscultural survey in 13 European countries. Value Health 2008;11(4):645-58.

17. Berra S, Bustingorryf V, Henze C, Díaz MP, et al. Adaptación transcultural del cuestionario KIDSCREEN para medir la calidad de vida relacionada con la salud en población argentina de 8 a 18 años. Arch Argent Pediatr 2009;107(4):307-14.
18. BerraS, TebéC,EsandiM,Carignano C. Fiabilidad y validez del cuestionario KIDSCREEN-52 para medir calidad de vida relacionada con la salud para población argentina de 8 a 18 años. Arch Argent Pediatr 2013;111(1):29-36.

19. Currie C,MolchoM, BoyceW,Holstein B, et al. Researching health inequalities in adolescents: the development of the Health Behaviour in School-Aged Children (HBSC) family affluence scale. Soc Sci Med 2008;66(6):1429-36.

20. Suldo SM, Riley KN, Shaffer E J. Academic Correlates of Children and Adolescents' Life Satisfaction. Sch Psychol Int 2006;27(5):567-82.

21. Palacio-Vieira JA, Villalonga-Olives E, Valderas JM, Espallargues M, et al. Changes in health-related quality of life (HRQoL) in a population-based sample of children and adolescents after 3 years of follow-up. Qual Life Res 2008;17(10):1207-15.

22. Villalonga-Olives E, Forero CG, Erhart M, PalacioVieira JA, et al. Relationship between life events and psychosomatic complaints during adolescence/youth: a structural equation model approach. J Adolesc Health 2011;49(2):199-205.

23. Santrock J. Psicología de la educación. México: McGraw Hill Interamericana; 2002.

24. Martínez Carpio H. Autopercepción social y atribuciones cognoscitivas en estudiantes de bajo rendimiento académico. Rev Electrón Investig Psicoeduc Psigopedag 2009;7(3):1175-216.

25. Casassus J, Cusato S, Froemel JE, Palafox JC, et al. Primer estudio internacional comparativo sobre lenguaje, matemáticas y factores asociados, para alumnos de tercer y cuarto grado de la educación básica. Informe técnico. Santiago: Laboratorio Latinoamericano de Evaluación de la Calidad de la Educación, UNESCO; 2001.

26. Jadue JG. Transformaciones familiares en Chile: Riesgo creciente para el desarrollo emocional, psicosocial y la educación de los hijos. Estud Pedagóg 2003;29:115-26.

27. Karvonen S, Vikat A, Rimpela M. The role of school context in the increase in young people's health complaints in Finland. J Adolesc 2005;28(1):1-16.

28. Piko BF. Self-perceived health among adolescents: the role of gender and psychosocial factors. Eur J Pediatr 2007;166(7):701-8. 